\title{
Palbociclib plus endocrine therapy in HER2 negative, hormonal receptor-positive, advanced breast cancer: A real-world experience
}

\section{Laura Pizzuti $^{1}$ | Antonio Giordano ${ }^{2}$ | Andrea Michelotti $^{3}$ | Marco Mazzotta ${ }^{4}$ | Clara Natoli $^{5}$ | Teresa Gamucci ${ }^{6,7}$ | Claudia De Angelis ${ }^{3}$ | Elisabetta Landucci ${ }^{3}$ | Lucrezia Diodati $^{3}$ | Laura lezzi $^{5}$ | Lucia Mentuccia $^{6}$ | Agnese Fabbri ${ }^{8}$ |} Maddalena Barba $^{1}$ | Giuseppe Sanguineti ${ }^{9}$ | Paolo Marchetti ${ }^{4,10}$ | Silverio Tomao ${ }^{11}$ | Luciano Mariani $^{12}$ | Ida Paris ${ }^{13}$ | Vito Lorusso ${ }^{14}$ | Simona Vallarelli ${ }^{14}$ | Alessandra Cassano ${ }^{15}$ | Francesca Airoldi ${ }^{15}$ | Armando Orlandi ${ }^{15}$ | Luca Moscetti ${ }^{16}$ | Domenico Sergi $^{1}$ | Maria Giuseppina Sarobba ${ }^{17}$ | Giuseppe Tonini ${ }^{18}$ | Daniele Santini $^{18}$ | Valentina Sini ${ }^{19}$ | Enzo Veltri ${ }^{20}$ | Angela Vaccaro ${ }^{5}$ | Laura Ferrari $^{5}$ | Michele De Tursi ${ }^{4}$ | Nicola Tinari ${ }^{4}$ | Antonino Grassadonia ${ }^{4}$ | Filippo Greco ${ }^{21}$ | Andrea Botticelli ${ }^{2}$ | Nicla La Verde ${ }^{22}$ | Claudio Zamagni ${ }^{23}$ | Daniela Rubino $^{23}$ | Enrico Cortesi ${ }^{24}$ | Valentina Magri ${ }^{24}$ | Giulia Pomati ${ }^{24}$ | Simone Scagnoli ${ }^{24}$ | Elisabetta Capomolla ${ }^{25}$ | Ramy Kayal ${ }^{26}$ | Angelo Fedele Scinto ${ }^{27}$ | Domenico Corsi $^{27}$ | Marina Cazzaniga ${ }^{28}$ | Lucio Laudadio ${ }^{29}$ | Samantha Forciniti ${ }^{29}$ | Maria Mancini $^{29}$ | Luisa Carbognin ${ }^{30}$ | Patrizia Seminara ${ }^{10}$ | Sandro Barni ${ }^{31}$ | Riccardo Samaritani $^{32}$ | Mario Roselli ${ }^{33}$ | Ilaria Portarena ${ }^{33}$ | Antonio Russo $^{34}$ (i) | Corrado Ficorella $^{35} \mid$ Katia Cannita ${ }^{35}$ | Silvia Carpano ${ }^{1}$ | Mirco Pistelli ${ }^{36}$ | Rossana Berardi $^{36}$ | Ruggero De Maria ${ }^{37}$ | Isabella Sperduti ${ }^{38}$ | Gennaro Ciliberto ${ }^{39}$ | Patrizia Vici ${ }^{1}$ (0)

\footnotetext{
${ }^{1}$ Division of Medical Oncology 2, IRCCS Regina Elena National Cancer Institute, Rome, Italy

${ }^{2}$ Department of Clinical and Molecular Medicine, "Sapienza" University of Rome, Azienda Ospedaliera Sant'Andrea, Rome, Italy

${ }^{3}$ UO Oncologia Medica I, Ospedale S. Chiara, Dipartimento di Oncologia, Dei Trapianti e Delle Nuove Tecnologie, Azienda Ospedaliera Universitaria Pisana, Pisa, Italy ${ }^{4}$ Department of Medical, Oral and Biotechnological Sciences, Centro Scienze Dell'Invecchiamento e Medicina Traslazionale-CeSI-MeT, Chieti, Italy

${ }^{5}$ Medical Oncology Unit, SS Trinità Hospital, Loc. San MarcianoHospital, Sora, Frosinone, Italy

${ }^{6}$ Medical Oncology Unit, SS Trinità Hospital, Sora, Italy

${ }^{7}$ Medical Oncology, Sandro Pertini Hospital, Rome, Italy

${ }^{8}$ Medical Oncology Unit, Belcolle Hospital, Viterbo, Italy
}

Abbreviations: $\mathrm{aBC}$, advanced breast cancer; Als, aromatase inhibitors; $\mathrm{CB}$, clinical benefit; CDK4/6, cyclin-dependent kinase 4/6; Cl, confidence interval; $\mathrm{CR}$, complete response; $\mathrm{ER}$, estrogen receptor; ET, endocrine therapy; HER2, human epidermal growth factor receptor 2; HR, hormone receptor; HRs, hazard ratios; LHRH, luteinizing hormone-releasing hormone; MBC, metastatic breast cancer; mTOR, mammalian target of rapamycin; N, number; OR, odd ratio; ORR, overall response rate; OS, overall survival; PFS, progression free survival; PgR, progesteron receptor; PI3K, phosphoinositide 3-kinase; PR, partial response; PS, performance status; RCTs, randomized clinical trials; RECIST, response evaluation criteria in solid tumors; SD, stable disease; Tam, tamoxifen. 
${ }^{9}$ Department of Radiation Oncology, IRCCS Regina Elena National Cancer Institute, Rome, Italy

${ }^{10}$ Department of Clinical and Molecular Medicine, A Oncology Division, "Sapienza” University of Rome, Rome, Italy

${ }^{11}$ Department of Clinical and Molecular Medicine, A Oncology Division, La "Sapienza" University of Rome, Rome, Italy

${ }^{12}$ HPV Unit, Department of Gynaecologic Oncology, IRCCS Regina Elena National Cancer Institute, Rome, Italy

${ }^{13}$ Gynecology Oncology Unit, Catholic University of the Sacred Heart, Rome, Italy

${ }^{14}$ Division of Medical Oncology, IRCCS, Giovanni Paolo II Hospital, Bari, Italy

${ }^{15}$ Department of Medical Oncology, Catholic University of Sacred Heart, Rome, Italy

${ }^{16}$ Division of Medical Oncology, Department of Oncology and Hematology, University Hospital of Modena, Modena, Italy

${ }^{17}$ Division of Medical Oncology, Ospedale San Francesco, Nuoro, Italy

${ }^{18}$ Department of Oncology, University Campus Biomedico of Rome, Rome, Italy

${ }^{19}$ Oncology Unit, ASL Roma 1, Santo Spirito Hospital, Rome, Italy

${ }^{20}$ Division of Medical Oncology, Ospedale S. Maria Goretti, Latina, Italy

${ }^{21}$ ASST Fatebenefratelli Sacco PO Fatebenefratelli, Department of Oncology, Milan, Italy

${ }^{22}$ Department of Oncology, ASST Fatebenefratelli Sacco PO Fatebenefratelli, Milan, Italy

${ }^{23}$ SSD Oncologia Medica “Addarii”, S. Orsola-Malpighi Hospital, Bologna, Italy

${ }^{24}$ Medical Oncology, Policlinico Umberto I, Sapienza, University of Rome, Rome, Italy

${ }^{25}$ Medical Oncology, Ospedale "Parodi-Delfino", Colleferro, Italy

${ }^{26}$ Department of Radiology, IRCCS Regina Elena National Cancer Institute, Rome, Italy

${ }^{27}$ Medical Oncology Unit, Ospedale San Pietro Fatebenefratelli, Rome, Italy

${ }^{28}$ Oncology Unit, ASST Monza, Monza, Italy

${ }^{29}$ Medical Oncology, Ospedale F. Renzetti, Lanciano, Italy

${ }^{30}$ Department of Pathology, Surgery and Oncology, "Mater Salutis" Hospital, ULSS21, Verona, Italy

${ }^{31}$ Department of Oncology, Oncology Unit, ASST Bergamo Ovest, Treviglio, Italy

${ }^{32}$ Medical Oncology Unit, "Regina Margherita" Hospital, Rome, Italy

${ }^{33}$ Medical Oncology Unit, Department of Systems Medicine, Tor Vergata Clinical Center University Hospital, Tor Vergata University Hospital, Rome, Italy

${ }^{34}$ Department of Surgical, Oncological and Oral Sciences, Section of Medical Oncology, University of Palermo, Palermo, Italy

${ }^{35}$ Medical Oncology Department, S. Salvatore Hospital, University of L'Aquila, L'Aquila, Italy

${ }^{36}$ Azienda Ospedaliero Universitaria Ospedali Riuniti Clinica di Oncologia, Università Politecnica delle Marche, Ancona, Italy

${ }^{37}$ Institute of General Pathology, Catholic University of the Sacred Heart, Rome, Italy

${ }^{38}$ Bio-statistics Unit, IRCCS Regina Elena National Cancer Institute, Rome, Italy

${ }^{39}$ Scientific Direction, IRCCS Regina Elena National Cancer Institute, Rome, Italy

\section{Correspondence}

Isabella Sperduti, Biostatistics Unit, IRCCS

Regina Elena National Cancer Institute, Via Elio Chianesi, 53-00144 Rome, Italy.

Email: isabella.sperduti@ifo.gov.it

Maddalena Barba, Division of Medical Oncology 2, IRCCS Regina Elena National Cancer Institute, Via Elio Chianesi,

53-00144 Rome, Italy.

Email: maddalena.barba@gmail.com

\section{Abstract}

Data from 423 human epidermal growth factor receptor 2-negative (HER2-), hormone receptor-positive $(\mathrm{HR}+)$ advanced breast cancer $(\mathrm{aBC})$ patients treated with palbociclib and endocrine therapy (ET) were provided by 35 Italian cancer centers and analyzed for treatment outcomes. Overall, 158 patients were treated in first line and 265 in second/ later lines. We observed 19 complete responses and 112 partial responses. The overall response rate (ORR) was 31\% (95\% confidence interval $[\mathrm{Cl}], 26.6-35.4)$ and clinical benefit was $52.7 \%$ (95\% Cl, 48-57.5). ORR was negatively affected by prior exposure to everolimus/exemestane $(p=0.002)$ and favorably influenced by early line-treatment $(p<0.0001)$. At 6 months, median progression-free survival was 12 months (95\% Cl, 8-16) and median overall survival was 24 months (95\% Cl, 17-30). More favorable outcomes were associated with palbociclib in early lines, no visceral metastases and no prior everolimus/exemestane. The main toxicity reported was neutropenia. Our results provide further support to the use of palbociclib with ET in HER2-, HR+ aBC. Differences in outcomes across patients subsets remain largely unexplained.

\section{KEYWORDS}

advanced breast cancer, hormonal therapy, endocrine resistance, palbociclib, real-world setting 


\section{1 | INTRODUCTION}

Approximately two-third of breast cancer patients are diagnosed with hormone receptor-positive ( $\mathrm{HR}+$ ) tumors. In advanced disease, endocrine therapy (ET) showed efficacy in more than a half of the patients. Unfortunately, both de novo and acquired resistance usually occur, making it particularly relevant research fostering, innovative treatments (Matutino, Joy, Brezden-Masley, Chia, \& Verma, 2018). Key components of cell cycle have been long investigated in breast and other cancer in the attempt to broaden our knowledge of the underlying biological mechanisms and orient research on therapeutic targets (Giordano et al., 1991, 1989; Roy, Sil, \& Chakraborty, 2018; Zhao et al., 2018).

Novel therapeutic strategies include the combination with agents targeting growth factors and angiogenesis and, more recently, specific targets such as the mammalian target of rapamycin (mTOR) pathway, phosphoinositide 3-kinase (PI3K), or cyclin-dependent kinase 4/6 (CDK4/6; D'Souza, Spicer, \& Lu, 2018).

Results from Phase 3 randomized trials have provided consistent support to the addition of CDK4/6 inhibitors (palbociclib, ribociclib, and abemaciclib) to an aromatase inhibitor in the first-line setting for most patients with human epidermal growth factor receptor 2 negative (HER2-), HR+, advanced breast cancer $(\mathrm{aBC})$ with substantial improvement in progression-free survival (PFS) and overall response rate (ORR) and manageable toxicity (Finn et al., 2016; Goetz et al., 2017; Hortobagyi et al., 2016). Recently, the combination of ribociclib and the selective estrogen receptor (ER) downregulator fulvestrant has shown high efficacy when used as first-line treatment (Slamon et al., 2018).

In patients recurring while on or soon after a previous adjuvant ET or while receiving first-line ET for advanced disease, the use of palbociclib, ribociclib, and abemaciclib, in combination with fulvestrant, is considered the standard of care (Cristofanilli et al., 2016; Slamon et al., 2018; Sledge et al., 2017), showing advantage in PFS and ORR. Subgroup analysis showed benefit with CDK4/6 inhibitors in all the subsets defined upon patientand tumor-related characteristics (Cristofanilli et al., 2016; Goetz et al., 2017; Hortobagyi et al., 2016; Slamon et al., 2018; Sledge et al., 2017). A clear advantage was seen even in premenopausal/ perimenopausal patients with the use of a luteinizing hormonereleasing hormone (LHRH) analogue (Goetz et al., 2017; Tripathy et al., 2018). Overall, quality of life was good (Harbeck et al., 2016) and toxicity manageable with asymptomatic neutropenia being the most common side effect and a very low incidence of febrile neutropenia (Kassem, Shohdy, Lasheen, Abdel-Rahman, \& Bachelot, 2018; Verma et al., 2016).

In 2015, the first CDK4/6 inhibitor developed, palbociclib, received accelerated US Food and Drug Administration approval for use in combination with the nonsteroidal competitive aromatase inhibitor letrozole for the treatment of postmenopausal HR+/HER2- $a B C$ as initial, endocrine-based therapy, and subsequently, in combination with fulvestrant in second line, in both premenopausal and postmenopausal patients. Since then, a considerable number of patients have been treated with palbociclib outside of randomized trials. The report from the expanded access of palbociclib/letrozole confirmed the clinical benefit, even in heavily pretreated patients (Brufsky et al., 2018). Moreover, some small reports of palbociclib administered in real-world practice seem to confirm efficacy and toxicity data, although these studies include a quite restricted number of patients (Chiu et al., 2017; Malik et al., 2017). In light of the renowned differences possibly emerging from the comparison between patients enrolled in randomized clinical trials (RCTs) and those from the realworld setting, we herein present additional evidence on the use of palbociclib plus an aromatase inhibitor or fulvestrant in HER2-, HR+, female $\mathrm{aBC}$ patients treated at several Italian cancer centers in reallife setting.

\section{2 | PATIENTS AND METHODS}

The primary objective of this study was to assess the clinical benefit and tolerability of palbociclib and ET (aromatase inhibitors or fulvestrant) as first or subsequent endocrine lines of therapy in patients with HER2-, HR+ aBC. Secondarily, we aimed to evaluate the efficacy in subgroups defined according to relevant patient- and tumor-related features.

Patients not enrolled in RCTs were retrospectively and sequentially identified and recruited at several Italian cancer centers. Palbociclib, in combination with ET, was given orally at $125 \mathrm{mg} /$ day, in 4-week cycles (3 weeks of treatment followed by 1-week off). Dose reductions/delay/discontinuations of palbociclib due to adverse events were recorded. Treatment was continued until disease progression, unacceptable toxicity, or patient refusal. Treatment efficacy was evaluated by Response Evaluation Criteria In Solid Tumors (version 1.1). Adverse events were recorded and graded according to the National Cancer Institute Common Terminology Criteria for Adverse Events (version 4.0).

All the patients signed a written informed consent to treatment and data collection. This study was conducted in accordance with the Declaration of Helsinki. The institutional ethic committees of the coordinating and satellite centers provided their approval to our study conduct.

\section{3 | STATISTICAL METHOD}

Descriptive statistics were computed for all the variables of interest. Continuous variables were presented as median and range. Proportions were exemplified by crude numbers and percentages. The Pearson $\chi^{2}$ test or the Fisher's exact test, when appropriate, were used to estimate the associations between categorical variables.

Survival curves were estimated by the Kaplan-Meier productlimit and the logrank test was used to assess differences between subgroups. Significance was defined at the $p \leq 0.05$ level. The impact of relevant clinical and pathological variables on the ORR and 
survival was tested in multivariate logistic models and Cox regressions. Multivariate logistic regression models and multivariate Cox proportional hazard models were developed using stepwise regression (forward selection). Variables testing significant at the univariate analysis were entered into the model, enter limit and remove limit were $p=0.10$ and 0.15 , respectively. The variables considered in univariate analysis included: age, Eastern Cooperative Oncology Group (ECOG) Performance Status (PS), stage at first diagnosis, line of treatment, histology, menopausal status, type and number of metastatic sites, treatment free interval, previous treatment with the inhibitor of mTOR everolimus, and fulvestrant pretreatment and dose reduction. The SPSS software (SPSS version 21.0, SPSS Inc. Chicago, IL) was used for all statistical evaluations.

\section{4 | RESULTS}

From September 2017 to June 2018, 423 advanced or metastatic HER2-, HR+ breast cancer patients were retrospectively identified and enrolled at 35 Italian cancer centers. Main patient and tumor characteristics are listed in Table 1. Median age was 60 years, with $10.4 \%$ of our patients being 75 years and older. The majority of our patients (337, 79.7\%) were postmenopausal, whereas 86 (20.3\%) were premenopausal, and received a LHRH analogue in combination with ET and palbociclib. Median ECOG PS was 0. Three hundred and one patients had ER-positive tumors, 279 patients had progesteron receptor (PgR)-positive tumors, and 274 patients had tumor expressing both ER and PgR, evaluated in breast tissue samples from primary tumors. In 182 patients, a biopsy of an amenable metastatic lesion was performed to confirm the HER2-/HR+ tumor status. The majority of our patients had visceral metastases (56.7\%); bone-exclusive disease was recorded in $18.9 \%$ of these patients, and $24.4 \%$ had multiple metastatic sites. One hundred and two patients (24.1\%) were metastatic at diagnosis.

\section{1 | Treatment received}

Among the 423 patients recruited, 158 (37.3\%) received palbociclibbased therapy as first-line treatment of advanced disease and 265 (62.7\%) as second and later lines (ranging from 1-11), including chemotherapy. Two hundred and seventy-five (65\%) patients had received prior adjuvant ET. Among them, $47.3 \%$ of patients had been treated with tamoxifen, $39.4 \%$ with an aromatase inhibitor, and $11.4 \%$ with tamoxifen for 2-3 years followed by an aromatase inhibitor for 2 to 3 years. Six patients had received unspecified adjuvant ET. One hundred and forty-nine patients (35.2\%) had recurred while on or within 12 months from the end of adjuvant ET. One hundred and eighty-eight (44.4\%) patients had received previous adjuvant chemotherapy followed by ET. Among the 265 patients treated with palbociclib for second and later lines, 224 patients had received at least one previous ET for advanced disease, whereas 41 patients had received only prior chemotherapy for metastatic disease, without ET. The majority of our patients had received an aromatase inhibitor, while 71 (26.8\%) patients had received fulvestrant and 84 (31.7\%) an everolimus-based treatment before palbociclib.

\section{2 | Efficacy}

All the patients included in this analysis were evaluable for efficacy (Table 2). We observed 19 (4.5\%) complete response (CR) and 112 (26.5\%) partial response (PR), for an ORR of 31\% (95\% confidence interval [CI], 26.6-35.4). Stable disease (SD) was reported in 175 (41.4\%) patients. A clinical benefit (CB), that is an objective response or SD lasting for at least 6 months, was observed in $52.7 \%$ of the patients ( $95 \% \mathrm{Cl}, 48-57.5)$. As concerns the line of treatment, when palbociclib-based treatment was administered as first line, we observed an ORR of $50.6 \%$ ( $95 \% \mathrm{Cl}, 42.8-58.4)$, and a CB of $70.2 \%$ (95\% Cl, 63.1-77.4). Among patients treated as first line, those recurred while on or within 12 months from the end of the adjuvant ET had an ORR of $35.9 \%(95 \% \mathrm{Cl}, 24.2-47.7)$ and a CB of $63 \%(95 \%$ $\mathrm{Cl}, 50.1-75.8)$. In the 102 patients with metastatic disease at diagnosis, the ORR was $32.4 \%(95 \% \mathrm{Cl}, 23.3-41.4)$ and the $\mathrm{CB}$ was $55.9 \%$ (95\% Cl, 46.2-65.5). In patients treated with palbociclib as second line of treatment, we observed 4 (3.8\%) CR and 20 (18.9\%) $\mathrm{PR}$, for an ORR of $22.7 \%(95 \% \mathrm{Cl}, 14.7-30.6)$. When palbociclibbased therapy was given as third or further treatment line, no CR was recorded and 27 (17\%) patients reached a PR, for an ORR of $17 \%$ (95\% Cl, 11.1-22.8). Among the 71 patients pretreated with fulvestrant, we observed 5 (7.1\%) CR and 16 (22.5\%) PR, for an ORR of $29.6 \%$ (95\% Cl, 19-40.2). A CB was reported in 35 (49.3\%) patients and progressive disease was observed in 26 (36.6\%) patients. Among the 84 patients pretreated with everolimus/ exemestane, we reported 1 (1.2\%) CR and 13 (15.5\%) PR, for an ORR of $16.7 \%$ (95\% Cl, 8.7-24.6). A CB was observed in 35 (41.7\%) patients and progressive disease in 40 (47.6\%) patients.

In our patient population, a statistically significant difference was observed in terms of ORR in patients pretreated with everolimus/exemestane versus patients who did not receive this latter treatment $(16.7 \%$ vs. $34.5 \%$, respectively, $p=0.002)$. Conversely, no statistically significant difference in ORR was observed according to previous fulvestrant $(31.7 \%$ vs. $29.6 \%, p=0.72)$. Both the ORR and the CB rate were higher in patients who did not have visceral metastases ( $p=0.0004$ and 0.04 , respectively, data available upon request). Treatment efficacy was not influenced by menopausal status, since ORR and $C B$ were similar between premenopausal and postmenopausal patients $(p=0.10$ and 0.1 , respectively).

In multivariate analysis including the overall population (Table 3), the only factor significantly related to ORR benefit was the administration of palbociclib-based treatment as early line-treatment $(p<0.0001)$. In the overall patient population, at a median follow-up of 6 months (95\% Cl, 2-28), median PFS was 12 months $(95 \% \mathrm{Cl}$, 8-16) and median overall survival (OS) was 24 months $(95 \% \mathrm{Cl}$, 17-30; Figure 1). Preliminary data regarding 1-year PFS and 1-year OS, estimated with Kaplan-Meier analysis, are shown in Table 4. Patients treated with palbociclib in early lines, without visceral 
TABLE 1 Main baseline characteristics of the study population $(N=423)$

\begin{tabular}{|c|c|}
\hline Characteristics & Patients, $N(\%)$ \\
\hline \multicolumn{2}{|l|}{ Age } \\
\hline Median (range) & $60(31-84)$ \\
\hline \multicolumn{2}{|l|}{ Menopausal Status } \\
\hline Post & $337(79.7)$ \\
\hline Pre & $86(20.3)$ \\
\hline \multicolumn{2}{|l|}{ Histology } \\
\hline Ductal & $334(79.0)$ \\
\hline Lobular & $68(16.1)$ \\
\hline Other & $21(5.0)$ \\
\hline \multicolumn{2}{|l|}{ Metastatic at diagnosis } \\
\hline Yes & $102(24.1)$ \\
\hline No & $321(75.9)$ \\
\hline \multicolumn{2}{|l|}{ Disease-free interval (months) } \\
\hline 0 & $102(24.1)$ \\
\hline$\leq 12$ & $149(35.2)$ \\
\hline$>12$ & $110(26.0)$ \\
\hline Unknown & $62(14.7)$ \\
\hline \multicolumn{2}{|l|}{ ECOG Performance Status ${ }^{a}$} \\
\hline 0 & $353(83.5)$ \\
\hline 1 & 53 (12.5) \\
\hline 2 & $17(4.0)$ \\
\hline \multicolumn{2}{|l|}{ Neoadjuvant chemotherapy } \\
\hline Yes & $55(13.0)$ \\
\hline No & $368(87.0)$ \\
\hline \multicolumn{2}{|l|}{ Adjuvant chemotherapy } \\
\hline Yes & $188(44.4)$ \\
\hline No & $235(55.6)$ \\
\hline \multicolumn{2}{|l|}{ Adjuvant hormonal therapy } \\
\hline None (metastatic disease at diagnosis) & $102(24.1)$ \\
\hline None (other reasons) & $46(10.9)$ \\
\hline Yes & $275(65)$ \\
\hline \multicolumn{2}{|l|}{ Type of adjuvant hormonal therapy ${ }^{b}$} \\
\hline Tamoxifen & $130(47.3)$ \\
\hline Letrozole & $51(18.5)$ \\
\hline Anastrozole & 45 (16.4) \\
\hline Exemestane & $12(4.4)$ \\
\hline Tam $\rightarrow$ Als & 31 (11.3) \\
\hline Unknown & $6(2.2)$ \\
\hline \multicolumn{2}{|l|}{ Adjuvant radiotherapy } \\
\hline Yes & $207(48.9)$ \\
\hline No & $216(51.1)$ \\
\hline \multicolumn{2}{|l|}{ Metastatic sites at palbociclib starting } \\
\hline Visceral & $240(56.7)$ \\
\hline Bone-only & $80(18.9)$ \\
\hline Other & $103(24.4)$ \\
\hline \multicolumn{2}{|l|}{ Number of metastatic sites } \\
\hline 1 & $150(35.4)$ \\
\hline 2 & $148(35.0)$ \\
\hline$\geq 3$ & $125(29.6)$ \\
\hline \multicolumn{2}{|l|}{ Previous treatment of $\mathrm{MBC}$} \\
\hline Yes & $265(62.6)$ \\
\hline No & $158(37.4)$ \\
\hline \multicolumn{2}{|l|}{ Previous chemotherapy for $\mathrm{MBC}$} \\
\hline None & $258(61.0)$ \\
\hline 1 line & 80 (18.9) \\
\hline 2 lines & $34(8.0)$ \\
\hline$\geq 3$ lines & $51(12.1)$ \\
\hline
\end{tabular}

(Continues)
TABLE 1 (Continued)

\begin{tabular}{|lc} 
Characteristics & Patients, $\mathbf{N}(\%)$ \\
Previous hormonal therapy for MBC & \\
None & $197(46.6)$ \\
1 line & $131(31.0)$ \\
2 lines & $56(13.2)$ \\
$>3$ lines & $39(9.2)$ \\
\hline
\end{tabular}

Note. Als: aromatase inhibitors; MBC: metastatic breast cancer; Tam: tamoxifen.

aPerformance status at palbociclib starting.

${ }^{b}$ Only for patients who underwent adjuvant endocrine therapy.

metastases and naïve to everolimus-based treatment seemed to have more favorable long-term outcomes.

In multivariate analysis, PFS was positively affected by lower ECOG PS $(p=0.001)$, no everolimus/exemestane pretreatment $(p=0.10)$, absence of visceral metastases $(p=0.01)$, and early treatment line $(p<0.0001)$, as described in Table 3 . In our patient population, palbociclib dose reductions did not influence PFS and OS ( $p=0.49$ and 0.63 , respectively).

\section{3 | Toxicity}

Data on toxicity are reported in Table 5. The main toxicity observed was hematological, with neutropenia of any Grade observed in 314 patients (74.2\%), being of Grade 3-4 in 183 patients (43.2\%). Neutropenic fever was extremely rare (3.5\%). No new safety issues emerged by our analysis. Palbociclib dose reduction was performed in 85 patients (20\%). Only one patient discontinued palbociclib treatment due to toxicity (persistent Grade 3-4 neutropenia). Extra-hematological toxicity was manageable with mild nausea and vomiting in $12.8 \%$ of these patients and mild to moderate fatigue observed in about half of our patients. No significant differences in toxicity were observed depending on palbociclib treatment line. In addition, no significant differences emerged when comparing toxicities across

TABLE 2 Best responses to palbociclib according to endocrine and/or chemotherapy treatment line $(N=423)$

\begin{tabular}{|c|c|c|c|c|}
\hline & \multicolumn{4}{|c|}{ Best response, $N(\%)$} \\
\hline & Overall & First line & Second line & $\begin{array}{l}\text { Third line } \\
\text { and beyond }\end{array}$ \\
\hline $\begin{array}{l}\text { Complete } \\
\text { response }\end{array}$ & $19(4.5)$ & $15(9.5)$ & $4(3.8)$ & $0(0)$ \\
\hline $\begin{array}{l}\text { Partial } \\
\text { response }\end{array}$ & $112(26.5)$ & $65(41.1)$ & $20(18.9)$ & $27(17.0)$ \\
\hline Stable disease & $175(41.4)$ & $51(32.3)$ & $61(57.5)$ & $63(39.6)$ \\
\hline $\begin{array}{l}\text { Progressive } \\
\text { disease }\end{array}$ & $117(27.6)$ & $27(17.1)$ & $21(19.8)$ & $69(43.4)$ \\
\hline Total & $423(100)$ & $158(100)$ & $106(100)$ & $159(100)$ \\
\hline $\begin{array}{l}\text { Overall } \\
\text { response rate }\end{array}$ & $131(31)$ & $80(50.6)$ & $24(22.7)$ & $27(17.0)$ \\
\hline $\begin{array}{l}\text { Clinical } \\
\text { benefit rate }\end{array}$ & $223(52.7)$ & $111(70.2)$ & $48(45.3)$ & $64(40.3)$ \\
\hline
\end{tabular}


TABLE 3 Multivariate analysis $(N=423)$

\begin{tabular}{|llll|}
\hline ORR & OR & $95 \% \mathrm{Cl}$ & $p$ \\
\hline Treatment line & - & - & $<0.0001$ \\
\hline 1st vs. 2nd & 3.50 & $2.02-6.08$ & $<0.0001$ \\
\hline 1st vs. >2nd & 5.04 & $2.99-8.42$ & $<0.0001$ \\
\hline 2nd vs. >2nd & 1.43 & $0.77-2.65$ & 0.25 \\
\hline PFS & HR & $95 \% \mathrm{Cl}$ & $p$ \\
\hline ECOG PS & & & \\
\hline 2 vs. 0-1 & 2.71 & $1.47-4.97$ & 0.001 \\
\hline Everolimus pretreatment & & & \\
\hline Yes vs. no & 1.42 & $0.94-2.16$ & 0.001 \\
\hline Visceral involvement & & & \\
\hline Yes vs. no & 1.66 & $1.13-2.44$ & 0.01 \\
\hline Treatment line & - & - & $<0.0001$ \\
\hline 2nd vs. 1st & 1.01 & $0.59-1.74$ & 0.97 \\
\hline >2nd vs. 1st & 2.33 & $1.43-3.80$ & 0.001 \\
\hline >2nd vs. 2nd & 2.31 & $1.39-3.82$ & 0.001 \\
\hline
\end{tabular}

Note. $\mathrm{Cl}$, confidence interval; ECOG PS: Eastern Cooperative Oncology Group Performance Status; HR: hazard ratio; OR: odd ratio; ORR: overall response rate; PFS: progression-free survival .

categories defined upon age, that is, $<75$ and $\geq 75$ years $(75.7 \%$ vs. $68.2 \%$, respectively; $p=0.28$; data available upon request).

\section{5 | DISCUSSION}

Palbociclib is a highly selective inhibitor of CDK4/6, indicated for the treatment of patients with HER2-/HR+, advanced or metastatic breast cancer, in combination with an aromatase inhibitor, as initial $\mathrm{ET}$, and with fulvestrant (plus LHRH analogue in premenopausal women) in patients previously treated with ET. In RCTs, palbociclib in combination with letrozole as first-line treatment in postmenopausal women (Finn et al., 2015, 2016) or in combination with fulvestrant in premenopausal, perimenopausal, or postmenopausal patients with progression after ET (Cristofanilli et al., 2016; Slamon et al., 2018; Sledge et al., 2017), significantly prolonged PFS and improved ORR. The benefit was significant in all prespecified subgroups. Further follow-up is needed to confirm the advantage in OS. Neutropenia was the most commonly reported any Grade and Grade 3 adverse event, with very low incidence of febrile neutropenia $(<2 \%)$. Hematologic toxicity is usually manageable, through dose delays, transient interruption, or dose reduction. However, these very encouraging data deriving from randomized trials need to be confirmed in realworld setting, in less-selected, and frail or more heavily pretreated patients.

The present retrospective observational study recruited 423 eligible female premenopausal and postmenopausal patients from 35 Italian oncologic centers and, to our knowledge, this is the largest case-series reported in the literature thus far. Overall, our study results are satisfactory, both in terms of efficacy and in terms of tolerability, and seem in line with those of the registrative trials (Cristofanilli et al., 2016; Finn et al., 2015, 2016; Harbeck et al., 2016; Verma et al., 2016) and other retrospective experiences. The results from the expanded access of palbociclib/letrozole in 126 USA patients showed encouraging data, with higher CB in first-line ET $(52.9 \%)$ than in subsequent lines (30.3\%), and a median PFS of 8.6 and 4.4 months in first line and endocrine pretreated patients, respectively. Moreover, the 24-month OS rate was $61.8 \%$ in patients with no prior exposure to $\mathrm{ET}$, being $39.8 \%$ in endocrine pretreated patients, and $63.1 \%$ in chemotherapy naïve, versus $31.8 \%$ in chemotherapy pretreated patients. The median OS was 19.8 months in endocrine pretreated patients and 14.9 months in patients having undergone prior chemotherapy. These results highlight the potential benefit of palbociclib-based combinations even in later lines of therapy (Brufsky et al., 2018).

A small trial from a single USA cancer center reports $26.5 \%$ of ORR in 22 patients treated with palbociclib and ET, with a median treatment duration of 5 months, an estimated PFS at 18 months of $50 \%$, and G3/4 neutropenia in $45 \%$ of the patients, with half of the patients requiring dose reductions (Malik et al., 2017). In a recent
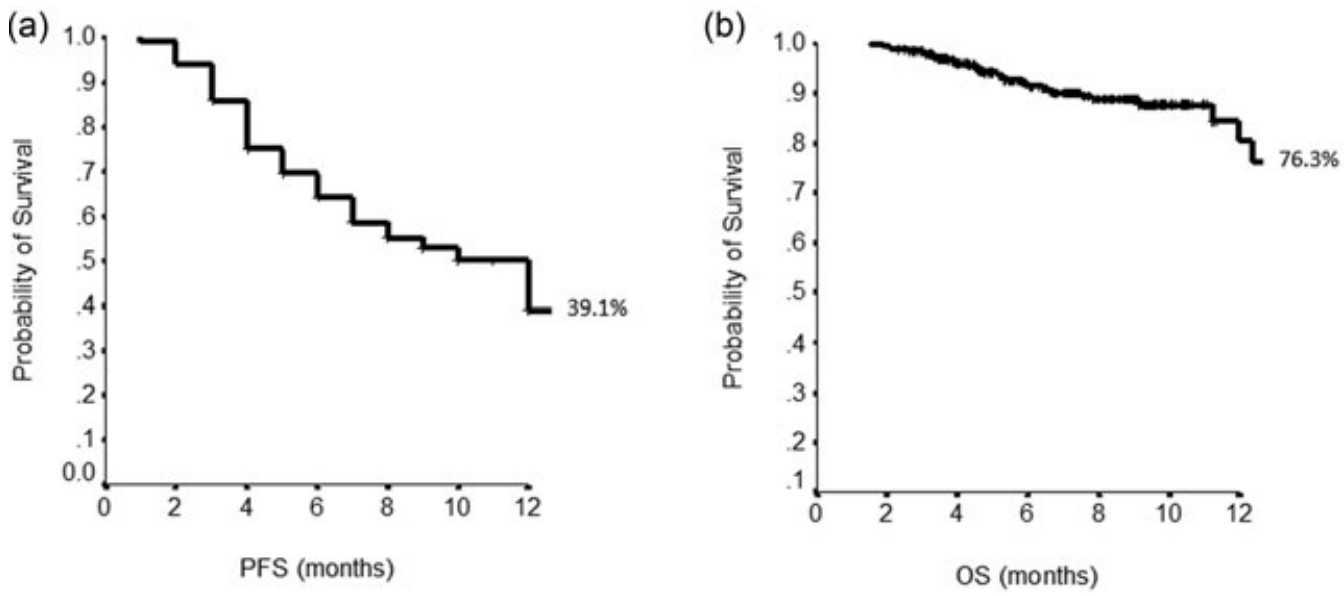

FIGURE 1 PFS (a) and OS (b) in the overall population. OS: overall survival; PFS: progression-free survival 
TABLE 4 One-year progression-free survival and 1-year overall survival according to patient characteristics $(N=423)$

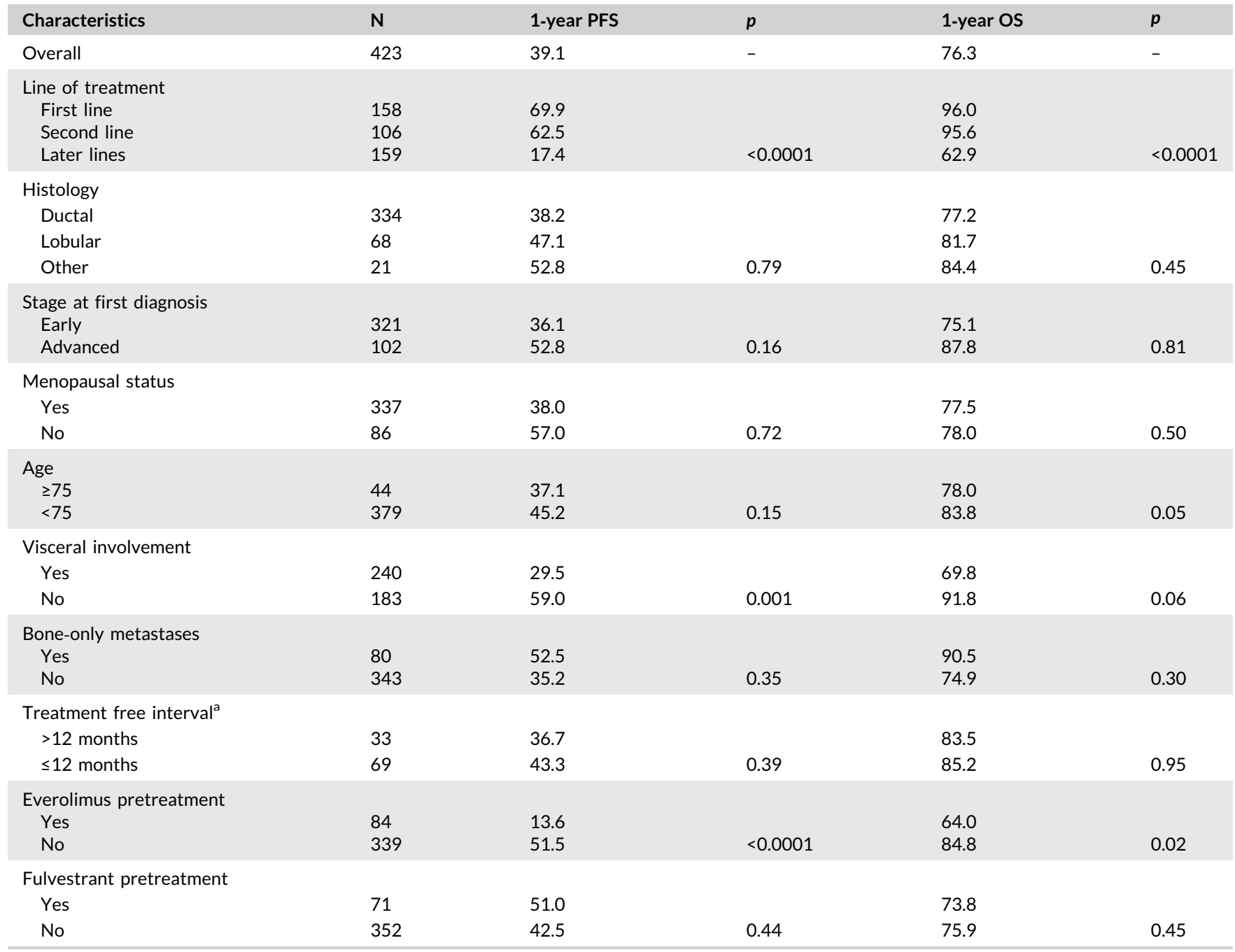

${ }^{a}$ From adjuvant endocrine treatment. Only for patients who received palbociclib as first line of treatment.

retrospective study, data from a US database of 763 patients treated with palbociclib and ET were analyzed. Six hundred and twelve of them received palbociclib in combination with letrozole. Mean follow-up was 6.4 months, and mean age 64 years. Dose reductions were reported in $20.1 \%$ of the patients, and $39.5 \%, 15.7 \%$, or $13.1 \%$ of the patients were treated as first, second, or later lines, respectively. Overall $74.6 \%$ of the patients had a neutropenic event, of Grade $3-4$ in $47.3 \%$ and $8.0 \%$ of these patients, respectively. No data on efficacy were reported (Kish et al., 2018). Data from a prospectively implemented database of 54 Chinese patients treated with a palbociclib in combination with aromatase inhibitor or fulvestrant in the real-world setting showed a disease control rate of $70 \%$ in 37 evaluable patients, $54 \%$ of response in patients with visceral site involvement, and a safety profile not dissimilar from that of the pivotal trials (Chiu et al., 2017). Another retrospective analysis in 24 heavily pretreated patients, treated with palbociclib and an aromatase inhibitor, showed a SD in $58.3 \%$ of patients, a median PFS of 4.8 months, and a median OS of 11 months. Treatment was well tolerated (Ban, Miše, Majić, Dražić, \& Vrdoljak, 2018). The compassionate use program in Belgium recruited 82 postmenopausal patients who received palbociclib and ET after at least 4 lines of systemic treatment. The median PFS was 3.17 months, with 34 patients not progressing within 6 months, resulting in an overall $C B$ of $41.5 \%$. The safety profile was favorable (Hoste et al., 2018).

Evidence from registrative trials confirms that palbociclib had similar had efficacy in nearly all prespecified subgroups (Finn, Crown, Ettl et al., 2016; Turner et al., 2018). Conversely, in our patient population, both ORR and CB rate were significantly higher in patients without visceral metastases. The results observed in visceral disease seem to translate into significant differences in long-term outcomes, since 1-year PFS is more favorable in patients without visceral involvement compared with patients with metastatic spread to viscera. However, caution is needed when interpreting results from subgroup analyses, given the restricted size and heterogeneity of the subsets compared in terms of disease and patients' characteristics.

As expected, the best outcome, both for 1-year PFS and 1-year OS, was observed when palbociclib was administered as first- and second-line, and no differences were observed according to menopausal status. An intriguing aspect of our study concerns the possibility of evaluating in real-life the efficacy of palbociclib in 
TABLE 5 Main toxicities in the study population $(N=423)$

\begin{tabular}{|c|c|c|c|c|}
\hline Toxicity & Grade $1(\%)$ & Grade 2 (\%) & Grade 3 (\%) & Grade 4 (\%) \\
\hline \multicolumn{5}{|l|}{ Hematologic } \\
\hline Leukopenia & 0.5 & 0.7 & 0.7 & - \\
\hline Neutropenia $^{a}$ & 10.4 & 20.6 & 37.1 & 6.1 \\
\hline Thrombocytopenia & 14.7 & 4.5 & 2.8 & - \\
\hline Anemia & 21.3 & 5.2 & 2.4 & - \\
\hline \multicolumn{5}{|l|}{ Nonhematologic } \\
\hline Nausea/vomiting & 12.8 & 1.4 & - & - \\
\hline Mucositis & 6.4 & 0.5 & 0.2 & - \\
\hline Diarrhea & 4.0 & 0.7 & 0.2 & - \\
\hline Constipation & 3.5 & 0.2 & 0.2 & - \\
\hline Abdominal pain & 4.5 & 0.7 & - & - \\
\hline Fatigue & 27.2 & 14.2 & 2.1 & - \\
\hline Hypertransaminasemia & 7.8 & 1.9 & 0.2 & - \\
\hline Anorexia & 2.4 & 0.2 & - & - \\
\hline Alopecia & 1.4 & 0.2 & - & - \\
\hline Headache & 0.7 & - & - & - \\
\hline Cutaneous toxicity & 2.1 & 0.5 & - & - \\
\hline Arthralgia & 0.9 & 0.5 & - & - \\
\hline QT prolongation & 0.5 & - & - & - \\
\hline
\end{tabular}

${ }^{\text {a }}$ Febrile neutropenia in 15 patients (3.5\%).

patients pretreated with fulvestrant or everolimus. In more detail, pretreatment with everolimus seems to negatively impact the efficacy of palbociclib, in terms of both ORR and long-term outcomes, whereas pretreatment with fulvestrant does not seem to negatively affect the activity of palbociclib-based treatment. Inherent data are scarcely available from randomized control trials in this setting, while some evidence has emerged from studies carried out in the realworld setting. Two hundred and six patients were treated with palbociclib and fulvestrant and/or everolimus in a French compassionate program. Almost a half of them received more than 6 lines, $48 \%$ of these patients had previously been treated with fulvestrant and $67.8 \%$ with everolimus in combination with ET. The median PFS of patients treated with fulvestrant and palbociclib was 5.46 months, with no significant differences between patients pretreated with fulvestrant or everolimus in univariate analysis (Arnedos et al., 2017). Within a compassionate use program, another single-centre retrospective analysis of 34 heavily pretreated patients showed a disease control rate of $52.9 \%$ at Week 12 and 24.4 at Week 24, and a PFS of 3.1 months, with no significant difference between everolimuspretreated (3.5 months) and everolimus-naïve patients (2.7 months; Maurer et al., 2018). Conversely, another small experience concerning 23 everolimus-pretreated patients showed a median PFS of 2.9 months, without objective responses and a CBR of $17.4 \%$, suggesting limited efficacy of palbociclib after progression with everolimus (Dhakal et al., 2018). This is consistent with our results from the present study. In another study, among 60 everolimus-pretreated patients, with 28 of them having received also fulvestrant and a median of 5 previous line of treatment, the median PFS was 5.8 months, and patients pretreated with fulvestrant had similar PFS, that is 6.4 months (Du Rusquec et al., 2018). Overall, these findings have to be confirmed by prospective, adequately sized clinical trials. Preclinical studies are also needed to specifically address the mechanisms leading to treatment failure in patients treated with ET plus CDK4/6 inhibitors following everolimus treatment. Results from these latter studies will help physicians determine the most appropriate sequence of hormonal therapies at an individual patient level.

In our study, the efficacy of palbociclib treatment was not affected by dose reductions, most frequently due to neutropenia. Our results are fully consistent with those from a retrospective report from the MD Anderson Cancer Center, which investigated the impact of dose delays and reductions on PFS in 334 patients receiving palbociclib in real-life setting (Clifton et al., 2017). In terms of safety, no new issues emerged in our patient population and no cumulative toxicity was reported. In addition, notwithstanding the quite restricted number of patients aged at least 75 years $(N=44)$, the present study supports the use of palbociclib in elderly patients, with no significant increase in toxicity observed by increasing age $(75.7 \%$ vs. $68.2 \%$, respectively; $p=0.28$; data available upon request).

Our study has some limitations. Although our results are consistent with those from the registrative trials and prior studies carried out in the real word setting, our follow-up is still particularly short and further data evaluation is needed following longer observation. The observational retrospective design deserves to be mentioned in light of the tendency towards confounding and biases which characterizes this type of studies and which invites caution in results' interpretation. In addition, as previously mentioned, data have come from 35 Italian cancer centers. The participation of such a remarkable number of cancer centers has provided our study with its greatest strength, that is, a sample size of 423 patients. At the same time, the lack of standardized operative procedures (SOPs) shared by each of the participating institutions diminishes somewhat our confidence in the chance of completely excluding heterogeneity in the biological and clinical assessment. However, at the single-centre level, SOPs are in place and quality controls are regularly performed relatively to the diagnostic workout. In addition, patients' therapeutic management and follow-up is in key with the good clinical practice. 
The strength of the present analysis is that it represents, to our knowledge, the largest cohort of palbociclib-based treatment in reallife setting, providing a considerable amount of data in support of the efficacy and tolerability of an innovative treatment in aBC outside of clinical trials. In these respects, the topic we addressed is of actual interest to a clinical research agenda. Our data also provide evidence concerning prior treatment with fulvestrant and/or everolimus, confirming no detrimental effect of fulvestrant pretreatment on palbociclib-based treatment outcome, whereas our results seem to suggest a less favorable outcome in everolimus-pretreated patients.

In conclusions, the development of CDK4/6 inhibitors and the introduction of palbociclib, the first agent of this class, into clinical practice certainly represent an important addition to the therapeutic armamentarium in HER2-, HR+ metastatic breast cancer. However several open questions remain concerning the role of CDK4/6 inhibitors in daily practice, such as the increased costs, the optimal place and sequence, the treatment beyond progression, and the identification of predictive markers. Data from the real word setting may have a confirmative role concerning the evidence on the efficacy of the treatment of interest in unselected patients' populations. At the same time, such data, notwithstanding the previously discussed limitations, may help reveal still unexplored traits of specific patients subsets, for example, patients previously exposed to everolimusbased treatment, whose characteristics may have not clearly emerged from prior RCTs and whose needs may remain unmet unless studies like ours take an appropriate place in the available literature.

\section{ACKNOWLEDGEMENTS}

This study was supported by Consorzio Interuniversitario Nazionale per la Bio-Oncologia (CINBO). We thank Ana Maria Edlisca and Dr. Rosa Carbone for editorial assistance and data managing.

\section{CONFLICTS OF INTEREST}

The authors declare that there are no conflicts of interest.

\section{AUTHOR CONTRIBUTIONS}

P. V., A. G., C. N., and T. G.: study conception and critical revision of the manuscript for important contents. T. G., L. P., M. M., M. B., I. S., and P. V.: manuscript drafting, study conduct, and data collection and analysis planning; A. M., M. M. S., G. S., P. M., S. T., R. D. M., and G. C. critically revised the manuscript for important intellectual content; I.S.: statistical analysis; C. D. A., E. L., L. D., L. I., L. M., A. F., I. P., V. L., S. V., A. C., F. A., A. O., L. M., D. S., G. S., G. T., D. S., V. S., E. V., A. V., L. F., M. D. T., N. T., A. G., F. G., A. B., N. L. V., C. Z., D. R., E. C., V. M., G. P., S. S., E. C., R. K., A. F. S., D. C., M. E. C., L. L., S. F., M. M., L. C., P. S., S. B., R. S., M. R., I. P., A. R., C. F., K. C., S. C., M. P., and R. B. data collection, database set up and implementation, and contribution to data analysis. All authors read and approved the final version of this manuscript and are responsible for all the aspects of the work.

\section{ORCID}

Antonio Russo (D) http://orcid.org/0000-0002-4370-2008

Patrizia Vici (iD http://orcid.org/0000-0001-7528-9783

\section{REFERENCES}

Arnedos, M., Rusquec, P., Morelle, M., Lebreton, C., Jacquet, E., \& Emile, G. (2017). Benefit from palbociclib and fulvestrant based on previous fulvestrant and/or everolimus treatment. Based on a cohort of over 200 patients treated in a French compassionate program. San Antonio Breast Cancer Symposium, P5-21-11.

Ban, M., Miše, B. P., Majić, A., Dražić, I., \& Vrdoljak, E. (2018). Efficacy and safety of palbociclib in heavily pretreated patients with HR+/HER2metastatic breast cancer. Future Oncology, 14, 537-544. https://doi. org/10.2217/fon-2017-0491

Brufsky, A., Davis, K., Mitra, D., Nagar, S., McRoy, L., \& Cotter, M. (2018). Retrospective assessment of treatment patterns and outcomes associated with palbociclib plus letrozole for postmenopausal women with HR+/HER2-advanced breast cancer enrolled in an early access program. San Antonio Breast Cancer Symposium, 78(4), Supplement.

Chiu, J., Leung, R., Sze, H., Teo, P., Choi, P., \& Lam, T. C. (2017). Real world data on use of palbociclib in hormone-receptor (ER) positive HER2 negative metastatic breast cancer (MBC) among Asian patients. Annals of Oncolology, 28(Issue suppl_10282017.)

Clifton, K. K., Kimmel, J., Yi, M., Chad, B., Litton, J., \& Debu, T. (2017). The impact of dose delays and reductions on toxicity and progression free survival (PFS) in patients receiving palbociclib. San Antonio Breast Cancer Symposium. Abstract P3-11-03.

Cristofanilli, M., Turner, N. C., Bondarenko, I., Ro, J., Im, S. A., Masuda, N., ... Slamon, D. (2016). Fulvestrant plus palbociclib versus fulvestrant plus placebo for treatment of hormone-receptor-positive, HER2-negative metastatic breast cancer that progressed on previous endocrine therapy (PALOMA-3): Final analysis of the multicentre, double-blind, phase 3 randomised controlled trial. Lancet Oncology, 17, 425-439. https://doi. org/10.1016/S1470-2045(15)00613-0

D'Souza, A., Spicer, D., \& Lu, J. (2018). Overcoming endocrine resistance in metastatic hormone receptor-positive breast cancer. Journal of Hematology and Oncology, 11(1), 80. https://doi.org/10.1186/s13045018-0620-6

Dhakal, A., Matthews, C. M., Levine, E. G., Salerno, K. E., Zhang, F., Takabe, K., ... Opyrchal, M. (2018). Efficacy of Palbociclib combinations in hormone receptor-positive metastatic breast cancer patients after prior everolimus treatment. Clinical Breast Cancer, 18(6):e1401e1405. https://doi.org/10.1016/j.clbc.2018.04.015

Finn, R. S., Crown, J. P., Ettl, J., Schmidt, M., Bondarenko, I. M., Lang, I., ... Slamon, D. J. (2016). Efficacy and safety of palbociclib in combination with letrozole as first-line treatment of ER-positive, HER2-negative, advanced breast cancer: Expanded analyses of subgroups from the randomized pivotal trial PALOMA-1/TRIO-18. Breast Cancer Research, 18, 67. https://doi.org/10.1186/s13058-016-0721-5

Finn, R. S., Crown, J. P., Lang, I., Boer, K., Bondarenko, I. M., Kulyk, S. O., ... Slamon, D. J. (2015). The cyclin-dependent kinase $4 / 6$ inhibitor palbociclib in combination with letrozole versus letrozole alone as first-line treatment of estrogen receptor-positive, HER2-negative, advanced breast cancer (PALOMA-1/TRIO-18): A randomised phase 2 study. Lancet Oncology, 16, 25-35. https://doi.org/10.1016/S14702045(14)71159-3

Finn, R. S., Martin, M., Rugo, H. S., Jones, S., Im, S. A., Gelmon, K., ... Slamon, D. J. (2016). Palbociclib and Letrozole in Advanced Breast Cancer. The New England Journal of Medicine, 375, 1925-1936. https://doi.org/10. 1056/NEJMoa1607303

Giordano, A., Lee, J., Scheppler, J., Herrmann, C., Harlow, E., Deuschle, U., ... Franza, B., Jr. (1991). Cell cycle regulation of histone H1 kinase 
activity associated with the adenoviral protein E1A. Science, 253(5025), 1271-1275.

Giordano, A., Whyte, P., Harlow, E., Franza, B. R., Jr., Beach, D., \& Draetta, G. (1989). A $60 \mathrm{kd}$ cdc2-associated polypeptide complexes with the E1A proteins in adenovirus-infected cells. Cell, 58(5), 981-990.

Goetz, M. P., Toi, M., Campone, M., Sohn, J., Paluch-Shimon, S., Huober, J., ... Di Leo, A. (2017). MONARCH 3: Abemaciclib as initial therapy for advanced breast cancer. Journal of Clinical Oncology, 35, 3638-3646. https://doi.org/10.1200/JCO.2017.75.6155

Harbeck, N., Iyer, S., Turner, N., Cristofanilli, M., Ro, J., André, F., ... Loibl, S. (2016). Quality of life with palbociclib plus fulvestrant in previously treated hormone receptor-positive, HER2-negative metastatic breast cancer: Patient-reported outcomes from the PALOMA-3 trial. Annals of Oncology, 27, 1047-1054. https://doi.org/10.1093/annonc/mdw139

Hortobagyi, G. N., Stemmer, S. M., Burris, H. A., Yap, Y. S., Sonke, G. S., PaluchShimon, S., ... O'Shaughnessy, J. (2016). Ribociclib as first-line therapy for HR-Positive, advanced breast cancer. The New England Journal of Medicine, 375, 1738-1748. https://doi.org/10.1056/NEJMoa1609709

Hoste, G., Punie, K., Wildiers, H., Beuselinck, B., Lefever, I., Van Nieuwenhuysen, E., ... Neven, P. (2018). Palbociclib in highly pretreated metastatic ER-positive HER-2 negative breast cancer. Breast Cancer Research and Treatment, 171, 131-141. https://doi.org/ 10.1007/s10549-018-4827-6

Kassem, L., Shohdy, K. S., Lasheen, S., Abdel-Rahman, O., \& Bachelot, T. (2018). Hematological adverse effects in breast cancer patients treated with cyclin-dependent kinase 4 and 6 inhibitors: A systematic review and meta-analysis. Breast Cancer, 25, 17-27. https://doi.org/10. 1007/s12282-017-0818-4

Kish, J. K., Ward, M. A., Garofalo, D., Ahmed, H. V., McRoy, L., Laney, J., ... Feinberg, B. A. (2018). Real-world evidence analysis of palbociclib prescribing patterns for patients with advanced/metastatic breast cancer treated in community oncology practice in the USA one year post approval. Breast CancerResearch, 20(1), 37. https://doi.org/10. 1186/s13058-018-0958-2

Malik, F., Ali, N., Jafri, S. I. M., Sundermeyer, M. L., Seidman, M. J., \& Fidler, C. J. (2017). Real world experience of palbociclib in hormone receptor-positive metastatic breast cancer. Journal of Clinical Oncology, 35(15_suppl) https://doi.org/10.1016/j.clbc.2018.09.00

Matutino, A., Joy, A. A., Brezden-Masley, C., Chia, S., \& Verma, S. (2018). Hormone receptor-positive, HER2-negative metastatic breast cancer: Redrawing the lines. Current Oncology, 25(Suppl 1), S131-S141. https://doi.org/10.3747/co.25.4000

Maurer, C., Ferreira, A. R., Martel, S., Lambertini, M., Pondé, N., Aftimos, P., ... Piccart, M. (2018). Endocrine therapy and palbociclib within a compassionate use program in heavily pretreated hormone receptorpositive, HER2-negative metastatic breast cancer. Breast, 39, 14-18. https://doi.org/10.1016/j

Roy, S., Sil, A., \& Chakraborty, T. (2018). Potentiating apoptosis and modulation of $\mathrm{p} 53, \mathrm{Bcl} 2$, and $\mathrm{Bax}$ by a novel chrysin ruthenium complex for effective chemotherapeutic efficacy against breast cancer. Journal of Cellular Physiology, https://doi.org/10.1002/jcp.27287.
Du Rusquec, P., Palpacuer, C., Campion, L., Patsouris, A., Augereau, P., Gourmelon, C., ... Frenel, J. S. (2018). Efficacy of palbociclib plus fulvestrant after everolimus in hormone receptor-positive metastatic breast cancer. Breast Cancer Research and Treatment, 168, 559-566. https://doi.org/10.1007/s10549-017-4623-8

Slamon, D. J., Neven, P., Chia, S., Fasching, P. A., De Laurentiis, M., Im, S. A., ... Jerusalem, G. (2018). Phase III randomized study of ribociclib and fulvestrant in hormone receptor-positive, human epidermal growth factor receptor 2-negative advanced breast cancer: MONALEESA-3. Journal of Clinical Oncology, 36(24), 2465-2472. https://doi.org/10. 1200/JCO.2018.78.9909

Sledge, G. W., Jr., Toi, M., Neven, P., Sohn, J., Inoue, K., Pivot, X., ... Llombart-Cussac, A. (2017). MONARCH 2: Abemaciclib in combination with fulvestrant in women with $\mathrm{HR}+/ \mathrm{HER} 2$-advanced breast cancer who had progressed while receiving endocrine therapy. Journal of Clinical Oncology, 35, 2875-2884. https://doi. org/10.1200/JCO.2017.73.7585

Tripathy, D., Im, S. A., Colleoni, M., Franke, F., Bardia, A., Harbeck, N., ... Lu, Y. S. (2018). Ribociclib plus endocrine therapy for premenopausal women with hormone-receptor-positive, advanced breast cancer (MONALEESA-7): A randomised phase 3 trial. Lancet Oncology, 19(7), 904-915. https://doi.org/10.1016/S1470-2045 (18)30292-4

Turner, N. C., Finn, R. S., Martin, M., Im, S. A., DeMichele, A., Ettl, J., ... Gelmon, K. A. (2018). Clinical considerations of the role of palbociclib in the management of advanced breast cancer patients with and without visceral metastases. Annals of Oncolology, 29, 669-680. https://doi.org/10.1093/annonc/mdx797

Verma, S., Bartlett, C. H., Schnell, P., Demichele, A. M., Loi, S., Ro, J., ... Rugo, H. S. (2016). Palbociclib in combination with fulvestrant in women with hormone receptor-positive/HER2-negative advanced metastatic breast cancer: Detailed safety analysis from a multicenter, randomized, placebo-controlled, Phase III study (PALOMA-3). TheOncologist, 21, 1165-1175. https://doi.org/10.1634/theoncologist. 2016-0097

Zhao, L., Jiang, L., He, L., Wei, Q., Bi, J., Wang, Y., ... Wei, M. (2018). Identification of a novel cell cycle-related gene signature predicting survival in patients with gastric cancer. Journal of Cellular Physiology, https://doi.org/10.1002/jcp.27365

How to cite this article: Pizzuti L, Giordano A, Michelotti A, et al. Palbociclib plus endocrine therapy in HER2 negative, hormonal receptor-positive, advanced breast cancer: A realworld experience. J Cell Physiol. 2018;1-10.

https://doi.org/10.1002/jcp.27832 